\title{
Determination of the direct band-gap energy of InAIAs matched to InP by photoluminescence excitation spectroscopy
}

\author{
P. Roura ${ }^{a)}$ \\ GRM, Dept. Enginyeria Industrial, Universitat de Girona, Av. Lluís Santaló s/n, E17071-Girona, \\ Catalonia, Spain \\ M. López-de Miguel, A. Cornet, and J. R. Morante \\ EME, Dept. Física Aplicada i Electrònica, Facultat de Física, Universitat de Barcelona, Av. Diagonal 647, \\ E08028-Barcelona, Catalonia, Spain
}

(Received 19 January 1996; accepted for publication 31 January 1997)

\begin{abstract}
A series of $\operatorname{In}_{x} \mathrm{Al}_{1-x} \mathrm{As}$ samples $(0.51<x<0.55)$ coherently grown on InP was studied in order to measure the band-gap energy of the lattice matched composition. As the substrate is opaque to the relevant photon energies, a method is developed to calculate the optical absorption coefficient from the photoluminescence excitation spectra. The effect of strain on the band-gap energy has been taken into account. For $x=0.532$, at $14 \mathrm{~K}$ we have obtained $E_{g 0}=1549 \pm 6 \mathrm{meV}$. (C) 1997 American Institute of Physics. [S0021-8979(97)00410-6]
\end{abstract}

\section{INTRODUCTION}

III-V semiconductors have a great technological interest in optoelectronics. In contrast to silicon, most of the materials in the III-V family have a direct band-gap that allows their use in electroluminescent devices. For this application, epitaxial layers of ternary and quaternary compounds are currently grown on GaAs or InP substrates. InAlAs is extensively used mainly for InAs compositions greater than 0.32 where its gap is direct. In particular, the $x=0.523$ composition is interesting because it corresponds to the material with its lattice parameter matched to that of InP; so, the precise knowledge of the band-gap energy for $x=0.523$ is relevant. Furthermore, when its value is known, the direct band-gap energy in the entire $x$ range can be calculated.

During the last 15 years, several works have afforded different values for the band-gap of the lattice-matched composition $^{1-5}$ at low temperatures. The values range from 1.443 up to $1.60 \mathrm{eV}$. This great scatter in the band-gap energy can be readily understood in view of the techniques used to measure the optical band-gap. Most studies rely on luminescence [photoluminescence (PL) ${ }^{1,3,4}$ or cathodoluminescence. $\left.{ }^{2}\right]$ However, as the luminescence at low temperature is dominated by impurity-related bands, a correction must be applied to obtain the optical band gap. This represents a major source of error because the ionization energies of the impurities are not known for InAlAs. The degree of uncertainty is illustrated with the series of high-quality InAlAs layers matched to InP analyzed in Ref. 3. The PL peak varied from 1.495 to $1.53 \mathrm{eV}$ in nominally equivalent layers. To avoid those difficulties, one should try to measure the optical absorption (OA) spectrum. However, as InP has a lower band-gap than InAlAs, it hides the InAlAs band-toband absorption. Alternatively, OA-related techniques can be applied. Following this approach, the work of Gaskill et al. ${ }^{5}$ is based on photoreflectance. Although in our opinion they give the best value reported to date, it was obtained by ex-

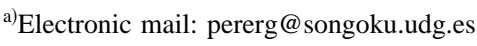

trapolation from the $80-400 \mathrm{~K}$ range and, additionally, it relies on one single sample.

Here we measure the band gap with a method based on photoluminescence excitation spectroscopy (PLE). As detailed below, although the InP substrate is opaque to the InAlAs direct gap region, we can determine the spectral shape of the InAlAs OA coefficient. The resulting spectra are fitted to obtain an accurate value of the band-gap. Besides being an independent determination of the band-gap energy, our method was applied to a series of samples ranging from tensed to compressed layers and the measurements were performed at low temperature $(14 \mathrm{~K})$. So, we avoid additional errors related to extrapolation and minimize the errors linked to scattering from sample to sample.

\section{EXPERIMENT}

The samples analyzed were InAlAs epitaxial layers grown on semi-insulating Fe-doped $\operatorname{InP}(100)$ substrates by molecular-beam epitaxy (MBE). The layers were not intentionally doped. Molar compositions were chosen to fall around the lattice matched composition $(x=0.523)$ (Table I). These values were obtained with a double-crystal x-ray diffractometer. A series of three reflections has been done for every sample. This procedure allowed the determination of the layer lattice parameters parallel to and perpendicular to the growth direction. Within the experimental accuracy, we ensured that the layers were coherently grown on the substrate without any strain relaxation. This strain (at $300 \mathrm{~K}$ ) is detailed in Table I.

The full width at half-maximum (FWHM) of the diffraction peaks was (for all samples except E) (Table I), below 100 arcsec, reaching the minimum for sample $\mathrm{C}$ (43 arcsec). These values reveal that the layers had a reasonable quality when compared with published results. The FWHM was usually greater than 50 arcsec (Refs. 1, 3 and 6) in strained samples unless the layers were grown over a buffer layer. ${ }^{6}$ The origin of the dispersion in the diffraction angle is the variation of the lattice parameter on a microscopic rather than a macroscopic scale. This was assessed by examining 
TABLE I. Sample characteristics.

\begin{tabular}{ccccc}
\hline \hline Sample & $\begin{array}{c}\text { Layer width } \\
(\mu \mathrm{m})\end{array}$ & $\begin{array}{c}\text { In molar } \\
\text { composition } x\end{array}$ & $\begin{array}{c}\text { Strain, } \epsilon \\
\left(\times 10^{-3}\right)\end{array}$ & $\begin{array}{r}\text { FWHM } \\
\text { diffraction } \\
(\operatorname{arcsec})\end{array}$ \\
\hline A & 1.2 & 0.512 & +0.66 & 74 \\
B & 3.0 & 0.516 & +0.45 & 97 \\
C & 1.2 & 0.519 & +0.38 & 43 \\
D & 1.09 & 0.539 & 1.14 & 55 \\
E & 2.19 & 0.552 & 1.94 & 130 \\
\hline \hline
\end{tabular}

various points on the layer surface. The diffraction peak shifted no more than $6 \mathrm{arcsec} / \mathrm{mm}$, which means that, as the $\mathrm{x}$-ray spot was of $6 \times 1 \mathrm{~mm}^{2}$, the corresponding FWHM would be less than 36 arcsec. On the other hand, from these measurements we have determined that the macroscopic variations of the molar composition are smaller than 0.0005 .

For optical measurements, samples were held at $14 \mathrm{~K}$ in a continuous-flow closed-circuit helium cryostat. In the PL experiments, the $488 \mathrm{~nm}$ line of an Ar laser was used for excitation. The luminescent emission was analyzed by a 0.6 $\mathrm{m}$ monochromator and detected with a GaAs photomultiplier. In the PLE experiments the excitation source was a 100 $\mathrm{W}$ halogen lamp coupled to the $0.6 \mathrm{~m}$ monochromator. A high-intensity low-resolution $0.2 \mathrm{~m}$ monochromator was used to detect the photons emitted. The PL and the PLE spectra were corrected by the transfer function and the lamp intensity, respectively.

\section{PL AND PLE SPECTRA}

The PL spectrum for each sample contained one peak. It is related to shallow donor and acceptor impurities, as usually occurs in low-temperature PL of III-V semiconductors. A typical PL spectrum is shown in Fig. 1. Although the energetic position is related to the band-gap energy, its large width linked to its complicated internal structure ${ }^{1}$ makes a fine determination of the band-gap energy impossible. So, we measured the PLE spectrum for every sample.

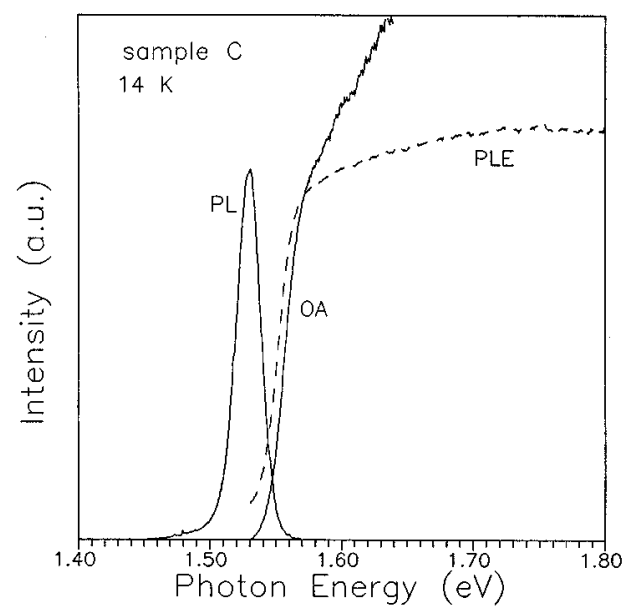

FIG. 1. Typical spectra obtained at low temperature. The OA spectrum is calculated from the PLE spectrum. Note the shift of the latter toward lower energies.

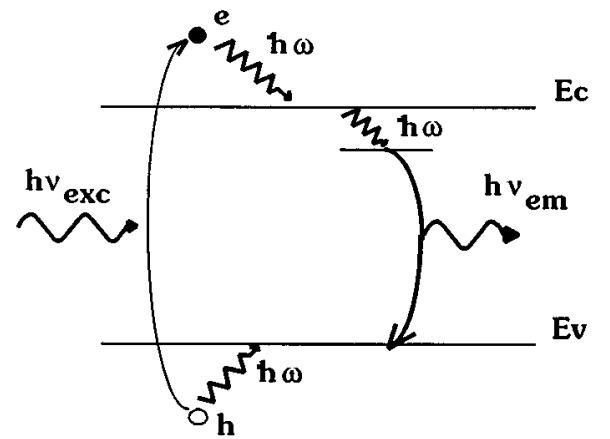

FIG. 2. Scheme of the excitation processes involved in the PLE spectrum.

As shown in Fig. 1 the PLE spectrum is a structureless band with a threshold on the low-energy side. After the threshold step the excitation increases smoothly and tends to an asymptotic value at high energies.

\section{ANALYSIS OF THE PHOTOEXCITATION MECHANISM}

We have found several studies ${ }^{6,7}$ that report PLE measurements on InAlAs. Our PLE spectra have the same spectral shape as those described in Ref. 6, which can be interpreted, in view of the detailed analysis performed on AlGaAs layers, ${ }^{8}$ as arising from the creation of electronhole pairs. Schematically, the processes involved are drawn in Fig. 2. In this section, we propose to analyze quantitatively the shape of the PLE spectra in order to obtain the optical absorption spectrum.

In a PLE experiment, the detection is fixed at the PL peak energy $\left(h \nu_{\mathrm{em}}\right)$ that involves states within the forbidden band. With the excitation monochromator, the exciting photon energy is scanned. When $h \nu_{\text {exc }}>E_{g}$ an electron-hole pair is created and, after quick thermalization, it can recombine radiatively through the impurity levels. This explains the threshold in the PLE spectrum that would be related to $E_{g}$.

According to this interpretation, the PLE spectrum contains valuable information about the optical absorption coefficient $\alpha(h \nu)$. In the case where nonradiative recombinations are negligible, the PLE intensity is proportional to the number of electron-hole pairs $\Delta n$, which, in turn, is proportional to the number of exciting photons absorbed by the epitaxial layer. We can write

$$
I_{\mathrm{PLE}} \propto \Delta n \propto\left(1-e^{-\alpha d}\right),
$$

where $d$ is the layer width. This relationship can be simplified in two situations. If the layer is very thin, we can expand the exponential function in first-order. This gives

$$
I_{\mathrm{PLE}} \propto \alpha d \propto \alpha .
$$

In this case, the PLE spectrum and $\alpha$ have the same shape. On the other hand, if the layer is thick enough, as $\alpha$ grows monotonically, the exponential will tend to zero asymptotically. So, we can write

$$
I_{\mathrm{PLE}}=I_{\infty}\left(1-e^{-\alpha d}\right),
$$




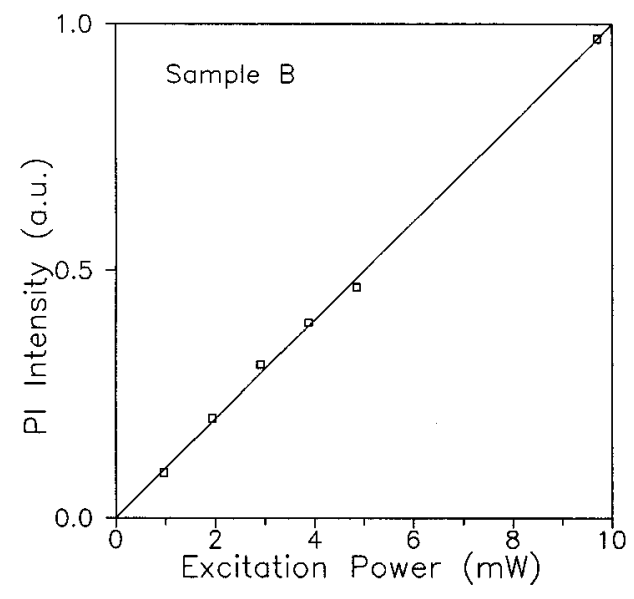

FIG. 3. Dependence of the PL intensity on the excitation power. The excitation source was, in this case, an argon laser.

where $I_{\infty}$ is the asymptotic value of $I_{\mathrm{PLE}}$ at high energies. In both thin and thick layers the absorption can be calculated from the PLE spectrum. In the second case a quantitative determination of the absorption coefficient is possible.

As mentioned above, formulas (2) and (3) are valid if the nonradiative recombinations are negligible. The same result would be obtained in the case where the nonradiative recombination rate were proportional to $\Delta n$. In both cases the PL intensity would be proportional to the excitation power. The experimental results agree with this expected behavior, as shown in Fig. 3.

\section{CALCULATION OF THE OA SPECTRA}

All PLE spectra show the asymptotic behavior toward $I_{\infty}$ predicted in the thick layer situation by Eq. (3) (Fig. 1). Accordingly, we have calculated the OA spectra (Fig. 4). In the case of sample A, the result is shown in Fig. 1. In the OA spectra the optical threshold is shifted to higher energies. If we take as reference the energy at half intensity of the

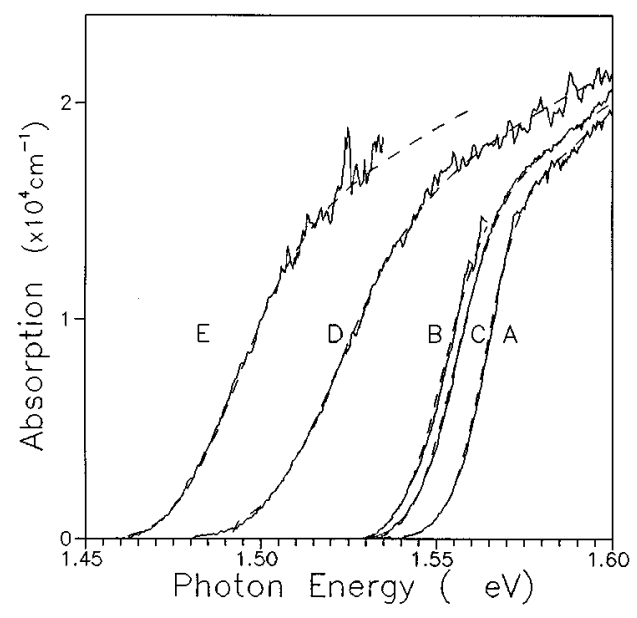

FIG. 4. Optical absorption spectra calculated from the PLE spectra. The result fits very well with the theoretical band shape (dashed line). Note that for every layer we obtain a similar absolute value of the absorption coefficient.
TABLE II. Elastic constants $\left(c_{11}, c_{12}\right)$ and deformation potentials $\left(b_{1}, b_{2}\right)$ of InAs and AlAs. The values for $\operatorname{In}_{0.5} \mathrm{Al}_{0.5} \mathrm{As}$ have been obtained by linear interpolation.

\begin{tabular}{lcccc}
\hline \hline & $\begin{array}{c}c_{11} \\
\left(\times 10^{11} \mathrm{dyn} / \mathrm{cm}^{2}\right)\end{array}$ & $\begin{array}{c}c_{12} \\
\left(\times 10^{11} \mathrm{dyn} / \mathrm{cm}^{2}\right)\end{array}$ & $\begin{array}{c}b_{1} \\
(\mathrm{eV})\end{array}$ & $\begin{array}{c}b_{2} \\
(\mathrm{eV})\end{array}$ \\
\hline $\mathrm{InAs}^{\mathrm{a}}$ & 8.329 & 4.526 & -5.79 & -1.8 \\
$\mathrm{GaAs}^{\mathrm{b}}$ & 12.02 & 5.70 & -7.96 & -1.5 \\
$\mathrm{In}_{0.5} \mathrm{Al}_{0.5} \mathrm{As}$ & 10.1 & 5.1 & -6.87 & -1.65 \\
\hline \hline
\end{tabular}

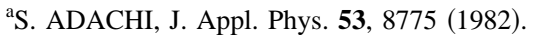

${ }^{\text {b }}$ S. Adachi, J. Appl. Phys. 58, R1 (1985).

threshold step, we find that the shift takes values between 4 and $10 \mathrm{meV}$. The highest values correspond to the thickest layers. This means that care has to be taken when the bandgap value is directly obtained from a PLE spectrum.

A test of self-consistency can be applied to the calculated spectra. As the molar composition is similar in all samples, the absolute value of the absorption coefficient must be the same. For all samples we have found $\alpha$ $=2.2-2.4 \times 10^{4} \mathrm{~cm}^{-1}$ at $E_{g}+100 \mathrm{meV}$. Furthermore, the matrix element of the optical transition is almost material independent within the III-V semiconductor family; ${ }^{9}$ so, $\alpha$ should be similar as well. For GaAs, ${ }^{10} \mathrm{InP},{ }^{11}$ and $\mathrm{In}_{0.5} \mathrm{Ga}_{0.5} \mathrm{As},{ }^{12} \alpha\left(E_{g}+100 \mathrm{meV}\right)$ takes the values of $1.4,1.5$, and $0.7 \times 10^{4} \mathrm{~cm}^{-1}$, respectively.

\section{DETERMINATION OF THE UNSTRAINED BAND-GAP ENERGY $\left(E_{g 0}\right)$}

Once the OA spectra have been obtained we can proceed to determine the band-gap energy. Samples are coherently grown on the InP substrate, so, the biaxial strain will modify the band structure: The valence band degeneracy is raised and the band-gap energy diminishes (increases) when the strain is tensile (compressive). Those effects can be calculated with the formula given in Ref. 13 and the material parameters of Table II. The valence-band splitting for $x$ $\approx 0.5$ is

$$
E_{\mathrm{LH}}-E_{\mathrm{HH}}=6.6 \epsilon(\mathrm{eV}),
$$

and the band-gap shift,

$$
\begin{aligned}
E_{g}-E_{g 0} & =+3.6|\epsilon|(\text { compressed layers }) \\
& =-10.2|\epsilon|(\text { tensed layers }),
\end{aligned}
$$

where $E_{g}$ is the value under strain. Due to the thermal expansion, the value of $\epsilon$ is slightly different at $14 \mathrm{~K}$ than at room temperature (Table I). From the thermal expansion coefficients of InAs, AlAs, and $\mathrm{InP},{ }^{14}$ the variation of $\epsilon$ can be estimated. It is found to be, approximately, $1.0 \times 10^{-4}$. Although the effect on the band-gap energy is very small $(0.4$ and $1 \mathrm{meV}$ for compressed and tensed samples, respectively), it has been taken into account. On the other hand, the thermal expansion would modify the band-gap splitting by no more than $1 \mathrm{meV}$ with a negligible effect on the band-gap energy.

The value of $E_{g}$ is obtained by fitting the OA spectra with the spectral shape given by Elliot. ${ }^{15}$ The procedure is described in detail in Ref. 16. Here we note that the internal 
TABLE III. Fitting parameters of the optical absorption spectra; valenceband splitting $\left(E_{\mathrm{LH}}-E_{\mathrm{HH}}\right)$; energy dispersion $\left(\sigma_{E}\right)$; exciton energy $\left(\epsilon_{x}\right)$; and band-gap energy $\left(E_{g}\right)$ (energies in $\mathrm{meV}$ ).

\begin{tabular}{crrrr}
\hline \hline Sample & $E_{\mathrm{LH}}-E_{\mathrm{HH}}$ & $\sigma_{E}$ & $\epsilon_{x}$ & $E_{g}$ \\
\hline $\mathrm{A}$ & +4.4 & 10.0 & 1.5 & 1563 \\
$\mathrm{~B}$ & +3.0 & 9.0 & 1.5 & 1554 \\
$\mathrm{C}$ & +2.5 & 9.0 & 1.5 & 1556 \\
$\mathrm{D}$ & -7.5 & 17.0 & 1.5 & 1519 \\
$\mathrm{E}$ & -12.8 & 18.0 & 1.0 & 1489 \\
\hline \hline
\end{tabular}

structure of the OA spectra consists of two bands arising from the valence-band splitting, which is known from the strain obtained by x-ray diffraction and formula (4). We have included this splitting although it is hidden due to the energy dispersion arising from microscopical inhomogeneities. The relevant fitting parameters are summarized in Table III. The accuracy of the determination of the band-gap energy due to the fitting procedure depends mainly on the energy dispersion $\left(\sigma_{E}\right.$ in Table III). We estimate that the error is smaller than \pm 3 and $\pm 2 \mathrm{meV}$ on the compressed and tensilestrained samples, respectively.

Finally, the unstrained band-gap energy can be calculated by using formula (5). The result is plotted in Fig. 5. Together with the experimental points, we have included, as a solid line, the expected variation of the band gap. This curve is determined by the binaries $\left[E_{g 0}(\operatorname{InAs})=0.418 \mathrm{eV}\right.$, $\left.E_{g 0}(\mathrm{AlAs})=3.13 \mathrm{eV}\right)^{14}$ and $E_{g 0}$ at $x=0.523$. As usual, a quadratic dependence on $x$ has been assumed. The value $E_{g 0}(x=0.523)$ has been chosen to give the best agreement with the experimental points. This method delivers the bandgap energy for the lattice matched composition: $E_{g 0}(x$ $=0.23)=1549 \pm 6 \mathrm{meV}$.

Apparently, the main sources of error are the macroscopic variation of the molar composition and the fitting accuracy. In the worst case, the associated error in the molar composition would be \pm 0.0005 . This error would be transmitted to the band gap through the strain [Eq. (5)]. However, this effect is negligible $( \pm 0.3 \mathrm{meV})$ compared with the estimated error of the fitting $( \pm 2 \mathrm{meV})$. In view of those val-

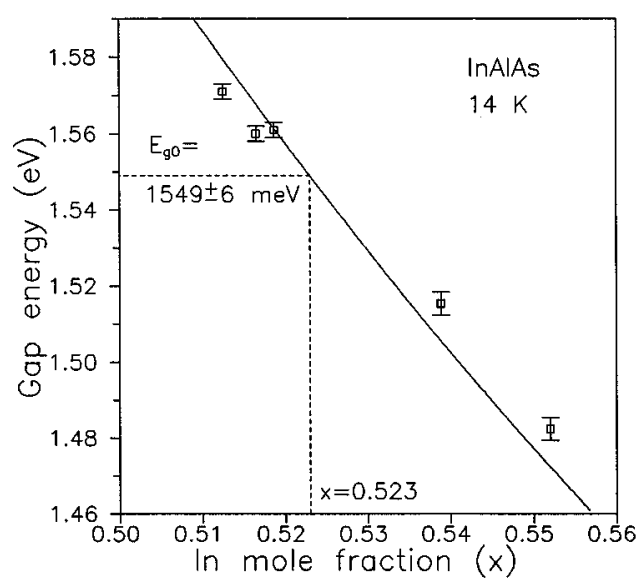

FIG. 5. Experimental values of the unstrained band-gap. The solid line is the parabola that takes the values of the binaris at $x=0$ and $x=1$. ues $\left(\Delta x= \pm 0.0005\right.$ and $\left.\Delta E_{g 0}= \pm 2 \mathrm{meV}\right)$ the dispersion of the experimental points in Fig. 5 is surprising. It is worth noting that in high-quality InGaAs/InP layers ${ }^{16}$ similar discrepancies between $\mathrm{X}$-ray diffraction and OA were encountered.

\section{SUMMARY AND CONCLUSIONS}

In the present article we have applied PLE spectroscopy to elucidate the value of the band-gap energy of InAlAs matched to InP at low temperature. As the InP substrate is opaque in the spectral region of interest, the optical absorption spectroscopy is not useful. In this case, the PLE spectroscopy is normally used instead. This can lead to significant errors as the optical threshold shifts to low energies when measured by PLE. These errors increase when the layers become thicker. To solve this problem, we have shown that, if the layers are thick enough, the optical absorption coefficient can be calculated from the PLE spectrum. The method also includes a self-consistency test.

The value for the lattice matched composition at $14 \mathrm{~K}$ is found to be $1549 \pm 6 \mathrm{meV}$. It compares quite well with the result obtained by photoreflectance by Gaskill et al. ${ }^{5}$ $\left(E_{g}=1541 \pm 2 \mathrm{meV}\right)$. This demonstrates that the techniques based on the intrinsic optical properties of InAlAs (reflectance or absorbance) are more suitable to determine the band-gap than PL, which usually requires uncertain corrections. We believe that the error bar of Gaskill et al. ${ }^{5}$ $( \pm 2 \mathrm{meV})$ is very optimistic if we consider that it is based on one single sample and that an extrapolation from $80 \mathrm{~K}$ was needed. In our previous work on InGaAs/InP epitaxial layers, ${ }^{16}$ the discrepancies between x-ray diffraction and optical absorption resulted in inaccuracies of $\pm 15 \mathrm{meV}$ even with samples that showed an energy dispersion low enough to allow a clear resolution of the valence-band splitting. The reader is also referred to the work of Bassignana, Miner, and Puetz $^{17}$ on InGaAs/InP, where the discrepancies between samples coming from different sources are analyzed.

This article represents a natural extension of previous studies devoted to the analysis of AO spectra in epitaxial layers $^{16,18}$ to the case where the substate is opaque. The most interesting possibility that arises is the evaluation of microscopic inhomogeneities. As shown in Ref. 18, the nature of the inhomogeneities (composition or strain variations) can easily be determined if the valence-band splitting is resolved.

\section{ACKNOWLEDGMENT}

This work was funded by the Spanish "Programa Ciencia de Materiales”' project under Contract No. MAT93-0564.

${ }^{1}$ D. Oertel, D. Bimberg, R. K. Bauer, and K.W. Carey, Appl. Phys. Lett. 55, 140 (1989).

${ }^{2}$ B. Wakefield, M. A. G. Halliwell, T. Kerr, D. A. Andrews, G. J. Davies, and G. J. Wood, Appl. Phys. Lett. 44, 341 (1984).

${ }^{3}$ L. Aina and M. Mattingly, Appl. Phys. Lett. 51, 1637 (1987).

${ }^{4}$ J. I. Davies, A. C. Marshall, M. D. Scott, and R. J. M. Griffiths, Appl. Phys. Lett. 53, 276 (1988).

${ }^{5}$ D. K. Gaskill, N. Bottka, L. Aina, and M. Mattingly, Appl. Phys. Lett. 56, 1269 (1990)

${ }^{6}$ E. Tournie, Y. Zhang, N. J. Pulsford, and K. Ploog, J. Appl. Phys. 70, $7362(1991)$. 
${ }^{7}$ J. P. Praseuth, L. Goldstein, P. Henoc, J. Primot, and G. Danan, J. Appl. Phys. 61, 215 (1987).

${ }^{8}$ E. F. Schubert and K. Ploog, J. Phys. C 18, 4549 (1985).

${ }^{9}$ H. Ehrenreich, Phys. Rev. 102, 1951 (1960).

${ }^{10}$ M. D. Sturge, Phys. Rev. 127, 768 (1962).

${ }^{11}$ W. J. Turner, W. E. Reese, and G. D. Pettit, Phys. Rev. 136, A1467 (1964).

${ }^{12}$ P. Roura, J. Bosch, S. A. Clark, F. Peiro, A. Cornet, and J. R. Morante, Semicond. Sci. Technol. 11, 1310 (1996).
${ }^{13}$ H. Asai, and K. Oe, J. Appl. Phys. 54, 2052 (1983).

${ }^{14}$ Landolt Börnstein, Numerical Data and Functional Relationships in Science and Technology (Springer, Berlin, 1982), Group III, Vol. 17.

${ }^{15}$ J. Elliot, Phys. Rev. 108, 1383 (1957).

${ }^{16}$ S. A. Clark, P. Roura, J. Bosch, A. Perez-Rodriguez, and J. R. Morante, J. Appl. Phys. 77, 3393 (1995).

${ }^{17}$ I. C. Bassignana, C. J. Miner, and N. Puetz, J. Appl. Phys. 65, 4299 (1989).

${ }^{18}$ P. Roura, J. Bosch, and J. R. Morante, Phys. Rev. B 46, 10453 (1992). 